\title{
Electro-assisted pretreatment of lignocellulosic materials in ionic liquid promoted organic solvents
}

\author{
Authorship: WANGQIYUE SUN ${ }^{\dagger}$, TAMAR L. GREAVES ${ }^{\ddagger}$, MAAZUZA Z. OTHMAN ${ }^{*}, \dagger$ \\ ${ }^{\dagger}$ School of Engineering, RMIT University, 124 La Trobe Street, Melbourne, Australia \\ ¥School of Science, RMIT University, 124 La Trobe Street, Melbourne, Australia \\ * Corresponding author: Maazuza.othman@rmit.edu.au
}

Wangqiyue Sun: wangqiyue.sun@rmit.edu.au

Tamar L. Greaves: tamar.greaves@rmit.edu.au,

Maazuza Othman: Maazuza.othman@rmit.edu.au

Mailing address of all authors: RMIT University, 124 La Trobe Street, Melbourne, Australia

\section{$\underline{\text { List of electronic supplementary information }}$}

Figure S1. The electrochemical colour change of [Emim]OAc/ethanol after CV scanning

Page S2

Figure S2. The FTIR spectra for untreated sawdust and post-EAOP sawdust

Page S3

Figure S3. The FTIR spectra for untreated rice straw and post-EAOP rice straw

Page S4

Figure S4. The FTIR spectra for untreated wheat straw and post-EAOP wheat straw.

Page S5

Figure S5. The ${ }^{13} \mathrm{C}$ NMR spectrum of the $[\mathrm{Bmim}] \mathrm{OAc} / \mathrm{GVL}$ before and post EAOP

Page S6 


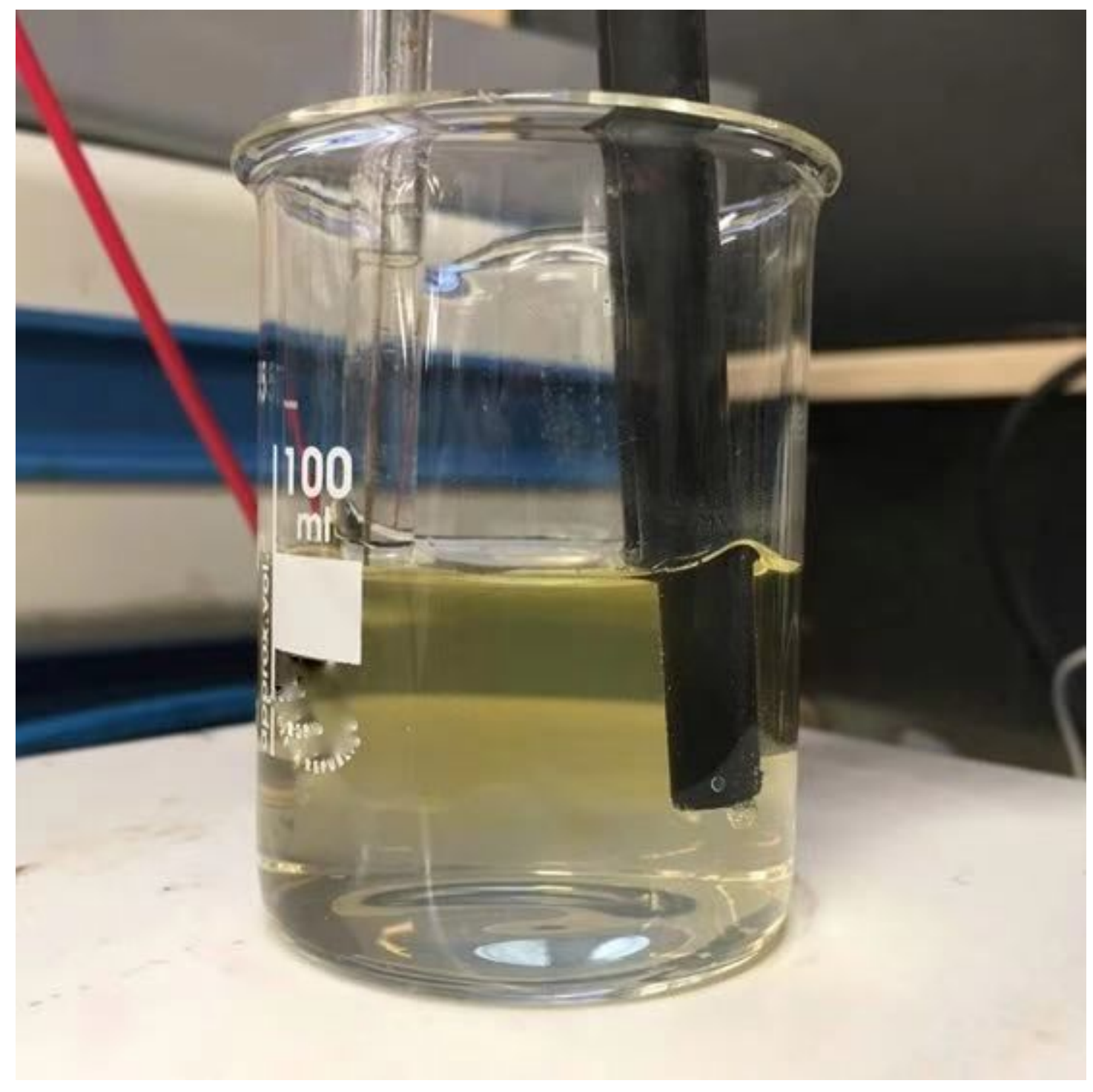

Figure S1. The electrochemical colour change of [Emim]OAc/ethanol after CV scanning. 


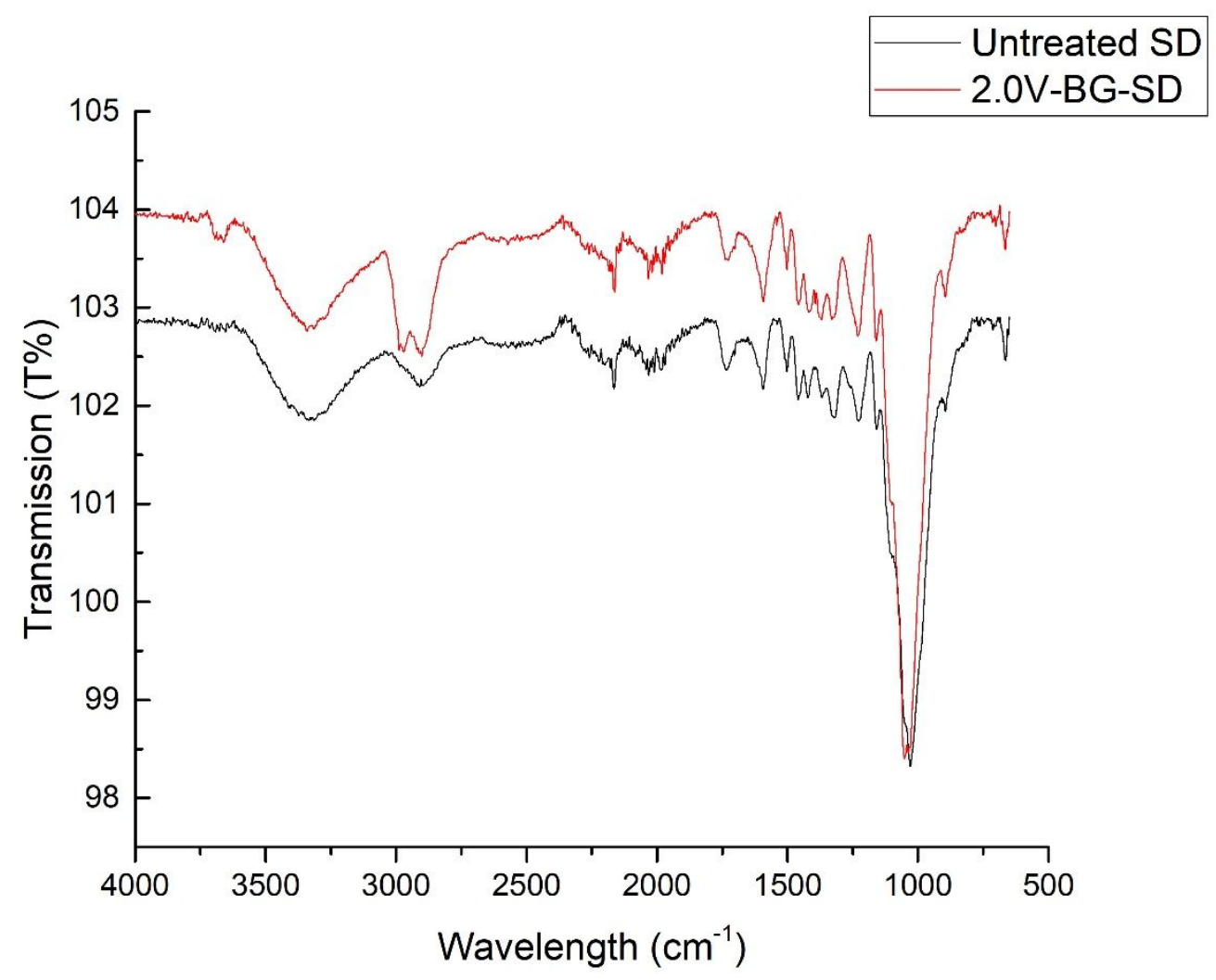

Figure S2. The FTIR spectra for untreated sawdust and post-EAOP sawdust. 


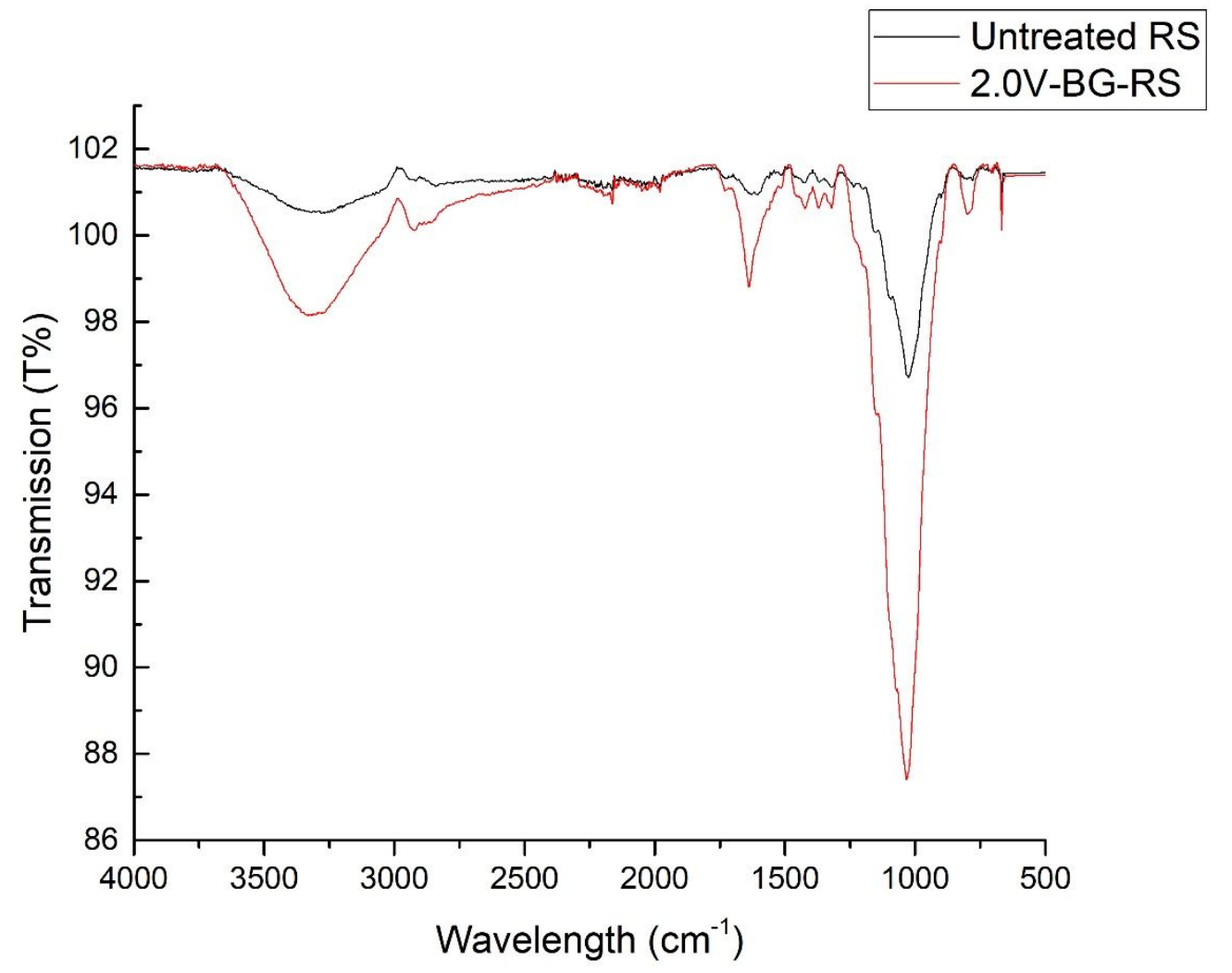

Figure S3. The FTIR spectra for untreated rice straw and post-EAOP rice straw. 


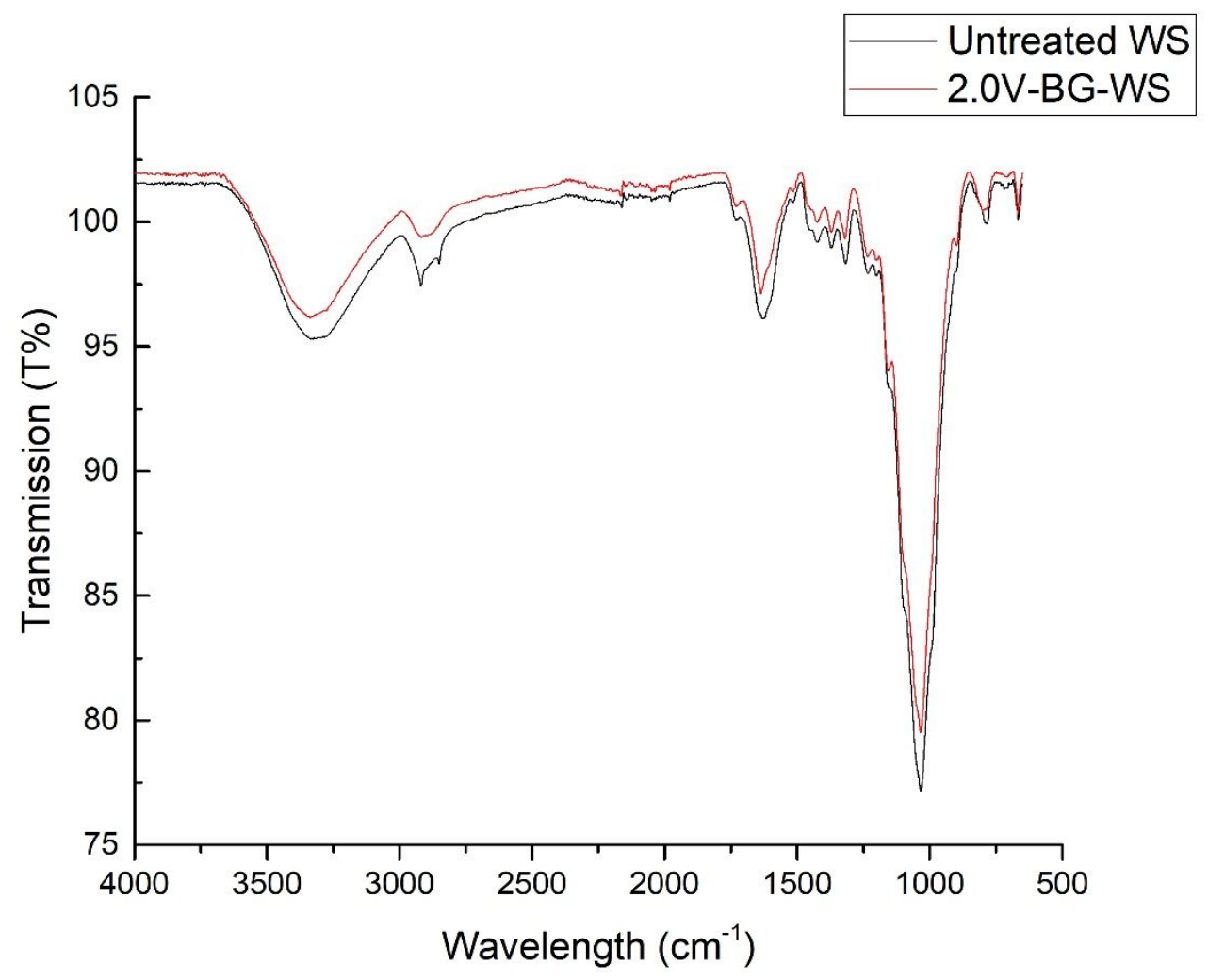

Figure S4. The FTIR spectra for untreated wheat straw and post-EAOP wheat straw. 


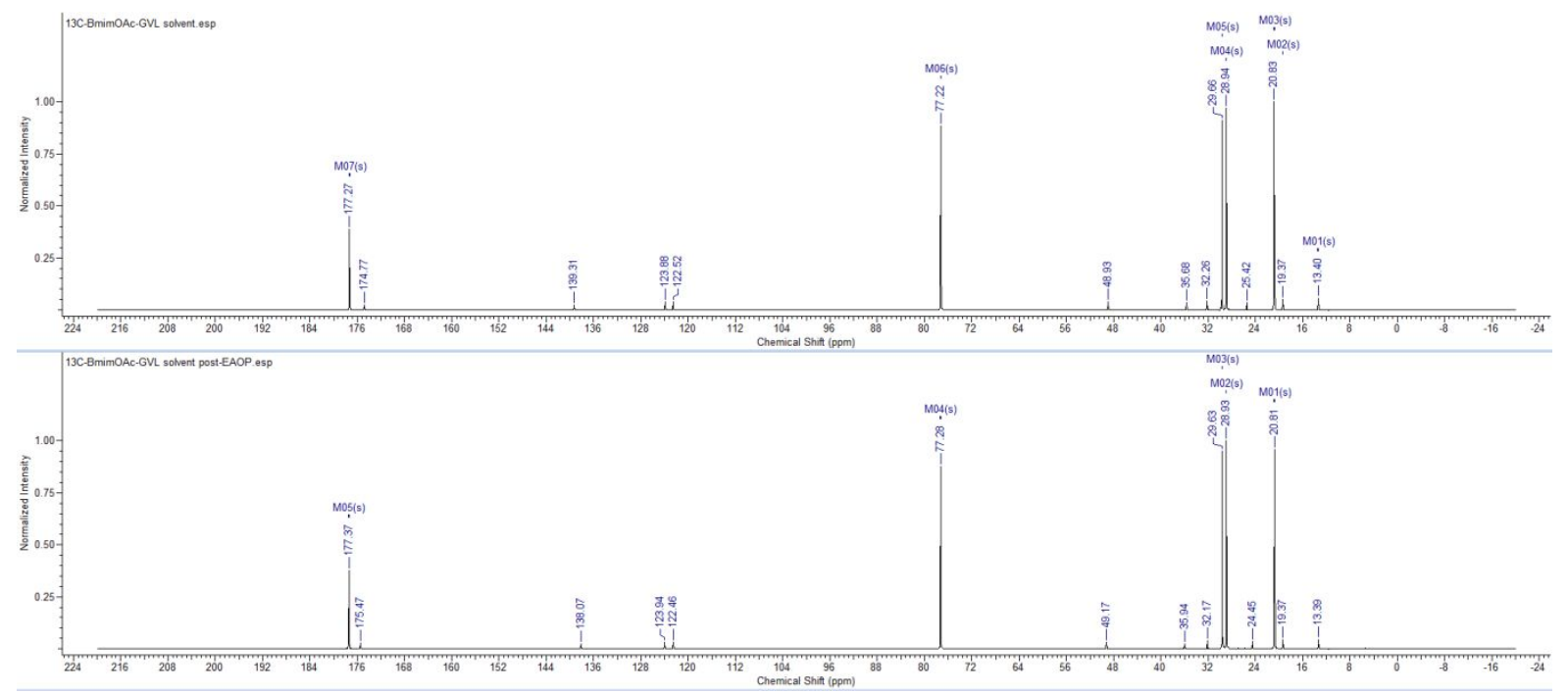

Figure $\mathrm{S} 5$. The ${ }^{13} \mathrm{C}$ NMR spectrum of the $[\mathrm{Bmim}] \mathrm{OAc} / \mathrm{GVL}$ before and post EAOP 